\title{
In-situ reduction study of anion concentration and its effects in the Fe-Ga-S system
}

\author{
Rebecca D. McAuliffe, Daniel P. Shoemaker \\ Materials Science and Engineering Department, University of Illinois at Urbana-Champaign
}

In-situ x-ray diffraction is a highly valuable technique that can be used to guide the synthesis of materials with emergent properties. This study uses in-situ x-ray diffraction in non-ambient conditions to understand the changes in anion concentrations in the Fe-Ga-S system during reduction reactions. This system allows for investigation into how anion concentrations affect emergent properties in ternary compounds by providing multiple layered antiferromagnetic ternary phases which can be synthesized with a range of sulfur content. By using in-situ x-ray diffraction, the lattice parameters of these phases are monitored throughout the reduction process. The contraction of the unit cell can be correlated with changes in lattice site occupancies giving insight into the reduction kinetics of these compounds. Once the reduction process is characterized, the experimental conditions for the synthesis of samples with varying anion concentrations can be determined from diffraction data. Samples are then synthesized and material properties, such as electronic transport and magnetism, are characterized to investigate the effect of anion content on these properties. A better understanding of how anion concentration affects material properties in the Fe-Ga-S system will help to build methods of studying reduction reactions as well as give insight into how electronic transport is affected by reduction conditions. 